\title{
ANÁLISE DE CENÁRIOS PARA IMPLANTAÇÃO DE UMA USINA DE RECICLAGEM DE RESÍDUOS DA CONSTRUÇÃO CIVIL NA REGIÃO AMAZÔNICA
}

\author{
Léo Daiki Shinomiya ' \\ Eduardo Junca ${ }^{2}$ \\ Felipe Fardin Grillo ${ }^{3}$ \\ Jefferson de Oliveira Gomes ${ }^{4}$ \\ Joner Oliveira Alves '
}

\section{Resumo}

A construção civil é uma das maiores atividades para o desenvolvimento econômico e social, contudo, também grande geradora de resíduos. Desta forma, a correta destinação dos resíduos é essencial para este segmento. Neste aspecto, o trabalho visou definir a melhor localização para a implantação de uma área de transbordo e triagem (ATT) e uma área de reciclagem (AR), em uma cidade da Região Amazônica, utilizando o método SMART (Simple Multi-Attribute Rating Technique). O método SMART é uma ferramenta que apoia o decisor com base em alternativas com multicritérios, possibilitando eficiência na avaliação e participação dos decisores (stakeholders) nas fases de desenvolvimento e decisão, proporcionando maior legitimidade do processo e compromisso na implementação dos resultados. Um estudo de caso foi desenvolvido para a região metropolitana de Belém-PA, tendo sido averiguado o bairro de São Brás como melhor local para implantação considerando, principalmente, o mínimo de área com vegetação e manancial atingida e logística facilitada dos resíduos.

Palavras-chave: Reciclagem; RCC; SMART; Análise de cenários.

\section{SCENARIO ANALYSIS FOR THE IMPLANTATION OF A CIVIL CONSTRUCTION WASTE RECYCLING COMPANY IN THE AMAZON REGION}

\begin{abstract}
Civil construction is one of the largest activities for economic and social development, but also a major waste generator. In this way, the correct disposal of waste is essential for this segment. In this aspect, the work aimed to define the best location for the implantation of a transshipment and sorting area (ATT) and a recycling area (AR), in a city of the Amazon Region, using the Simple Multi-Attribute Rating Technique (SMART). The SMART method is a tool that supports the decision maker based on alternatives with multiple criteria, allowing efficiency in the evaluation and participation of the stakeholders in the development and decision phases, providing greater legitimacy of the process and commitment in the implementation of the results. A case study was developed for the metropolitan area of Belém-PA, and the São Brás neighborhood was investigated as the best site for implantation considering, mainly, the minimum area with vegetation and wells reached and facilitated logistics of the residues.
\end{abstract}

Keywords: Recycling; Waste construction; SMART; Scenario analysis.

\footnotetext{
'Instituto SENAI de Inovação em Tecnologias Minerais - ISI-TM, Federação das Indústrias do Estado do Pará - FIEPA, Belém, PA, Brasil. 2Universidade do Extremo Sul Catarinense - UNESC, Criciúma, SC, Brasil.

${ }^{3}$ Instituto Federal de Educação, Ciência e Tecnologia do Espirito Santo - IFES, Vitória, ES, Brasil. E-mail: felipefarding@gmail.com

${ }^{4}$ Instituto Tecnológico de Aeronáutica - ITA, São José dos Campos, SP, Brasil.
}

2176-I523 (C) 2019 Associação Brasileira de Metalurgia, Materiais e Mineração. Published by ABM. This is an open access paper, published under the Creative Commons CC BY-NC-ND license (Attribution-NonCommercial-NoDerivs) - https://creativecommons.org/licenses/ by-nc-nd/4.0/. 


\section{INTRODUÇÃO}

A construção civil é um setor que contribui para o papel econômico quanto para o papel social do País, e, ao mesmo tempo grande geradora de resíduos. A quantidade de entulho gerado corresponde, em média, a $50 \%$ do material desperdiçado, sendo que no Brasil são produzidos cerca de 850.000 t/mês [I]. Na região do Pará ainda não existe um sistema de tratamento de resíduos sólidos da construção civil (RCCs) organizado pelo governo, apenas empresas particulares. Estes resíduos, na maioria das vezes, são depositados em locais irregulares, como terrenos baldios [2].

Segundo a Política Nacional de Resíduos Sólidos [3], todos os geradores são responsáveis pelos seus resíduos, seja na execução de uma pequena reforma residencial ou na construção de um edifício. Assim, a correta disposição passa pelo recolhimento e processamento destes resíduos em uma usina de beneficiamento para transformar a matéria bruta em um produto para outras cadeias, de maneira sustentável e com um menor custo e tempo. Também é necessário a concentração em um local estratégico os resíduos para transbordo e triagem [4]. A ABNT NBR $n^{\circ}$ I5. I I 2c [5] fixa os requisitos exigíveis para este tipo de projeto, a implantação e a operação da área de transbordo e triagem de resíduos da construção civil e resíduos volumosos. Enquanto que, a ABNT NBR $n^{\circ}$ I 5.1 | 4a [6] fixa os requisitos para a instalação e operação da área de reciclagem.

As propostas de produtos que surgirem neste contexto devem atender a princípios como: basear-se fundamentalmente em recursos renováveis, garantindo ao mesmo tempo sua renovação; aperfeiçoar o emprego dos recursos; não acumular lixo no ecossistema; garantir o direito ao gozo do espaço ambiental [7]. O objetivo deste trabalho foi avaliar a melhor localização estratégica para a captação e o beneficiamento de RCC composto por uma área de transbordo e triagem (ATT) e uma área de reciclagem (AR) utilizando o método SMART (Simple Multi-Attribute Rating Technique).

\section{MATERIAIS E MÉTODOS}

O método SMART é utilizado quando o decisor se depara com uma situação que deve ser solucionada entre uma série de alternativas conflitantes e concorrentes. Neste caso duas opções básicas se apresentam: usar intuição gerencial e realizar uma modelagem da situação, de maneira a estudar mais profundamente o problema [4]. O método SMART pode ser definido em três fases (Figura I). A I a fase é composta pela estrutura do problema com a definição dos objetivos e decisores. Na fase 2 estrutura-se o MCDA (Multi-Criteria Decision Analysis - Método de decisão multicritério), no qual um conjunto de metodologias de apoio à tomada de decisões com critérios definidos são considerados simultaneamente e de forma explícita. A fase 3 apresenta um estudo sobre as avaliações das alternativas derivadas das fases anteriores $[8,9]$.

Este método também pode auxiliar no processo de solucionar problemas com a participação dos stakeholders e decisores no desenvolvimento, dando maior legitimidade do processo e compromisso na implementação dos resultados.

Com relação à destinação dos $\mathrm{RCCs}$, esta é de responsabilidade de seu gerador, incluindo ações voltadas a seu reuso, reciclagem ou destinação responsável [3]. Para o presente trabalho, foi considerado que a usina buscará os resíduos nas construções, não eximindo a responsabilidade da mesma, e sim incentivando-as a retrabalharem o RCC de maneira mais apropriada e que possa gerar menor impacto ao meio ambiente. Considerando o objetivo de empregar o método SMART para análise de cenários da implantação de uma ATT/AR em uma cidade da Região Amazônica, um estudo de caso foi desenvolvido para a região metropolitana de Belém-PA.

\section{Fase 1: Estruturação do Problema}

Passo 1: Definição dos objetivos e Decisores

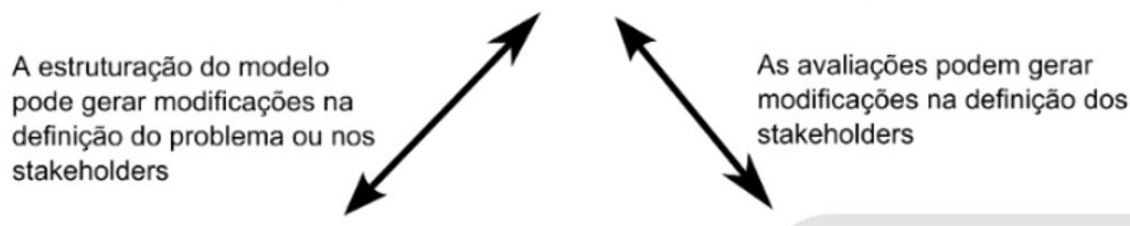

Fase 2: Estruturação do Modelo MCDA

Passo 2: Contrução da Árvore de Valor

Passo 3: Identificação de Alternativas

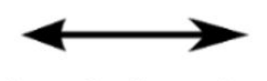

As avaliações podem gerar modificaçöes na estrutura do modelo
Fase 3: Avaliação das Alternativas Passo 4: Construção das Funções de Valor Passo 5: Avaliação parcial das Alternativas Passo 6: Definição os pesos dos atributos Passo 7: Avaliação global das alternativas Passo 8: Avaliação da decisão

Passo 9: Realização da Análise de Sensibilidade

Figura I. Fases de aplicação do modelo SMART. Adaptado de Franco e Montibeller [8]. 


\section{RESULTADOS E DISCUSSÃO}

Os resultados e discussões para o problema proposto estão apresentados de acordo com as fases de aplicação do modelo SMART (Figura I), conforme a seguir.

\section{I Passo I: Definição dos Objetivos e Decisores}

Os principais stakeholders foram identificados como: donos de construtoras, a sociedade, as empresas de demolição, as empresas para atividade fim. E para o grupo de decisores foram consideradas a SEMMA (Secretaria Municipal de Meio Ambiente), SEMA (Secretaria e Estado de Meio Ambiente), as empresas para atividade fim, os donos das construtoras e especialistas.

Um brainstorming foi realizado durante uma reunião do Conselho Temático de Meio Ambiente e Sustentabilidade (COEMA) da Federação das Indústrias do Estado do Pará (FIEPA) visando identificar as principais ideias dos stakeholders e decisores para a escolha do melhor local para implantar a usina. As ideias principais deste processo foram: minimizar o gasto de insumos; minimizar o impacto ambiental; minimizar área que contenha vegetação e hidrografia; minimizar o custo do terreno; maximizar proximidade das construtoras; maximizar a logística de pessoas e de RCC.
Em seguida, foram definidos os objetivos meios e fins. Para os objetivos fins foram considerados: maximizar a logística; minimizar o impacto ambiental; minimizar o custo. Para os objetivos meios foram considerados: minimizar o gasto de insumos; maximizar a logística de pessoas e de RCC; maximizar o rendimento dos equipamentos; maximizar proximidade das construtoras; minimizar área que contenha vegetação e hidrografia; minimizar o custo do terreno; minimizar a emissão de $\mathrm{CO}_{2}$.

\subsection{Passo 2: Construção da Árvore de Valor}

A estruturação do problema foi iniciada com a realização de um brainstorming com as principais ideias registradas na Figura 2.

Então buscou-se atributos para mostrar valores quantitativos e qualitativos sobre a situação local, onde se definiu os critérios e os subcritérios (Figura 3). Neste trabalho os critérios internos da usina como exemplo, o critério produtividade, não foram levados em consideração devido não influenciarem na escolha da melhor localização da usina, em um estudo posterior pretende-se considerar fatores internos que influenciam na saúde dos trabalhadores e da empresa.

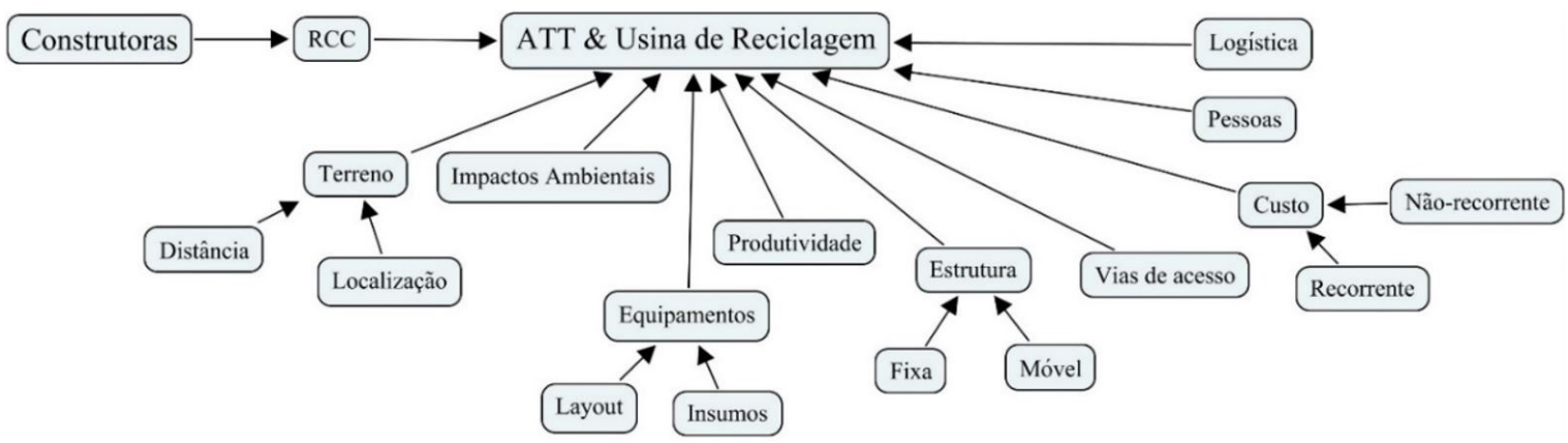

Figura 2. Requisitos de uma ATT e de uma Usina de reciclagem definidos através do brainstorming.

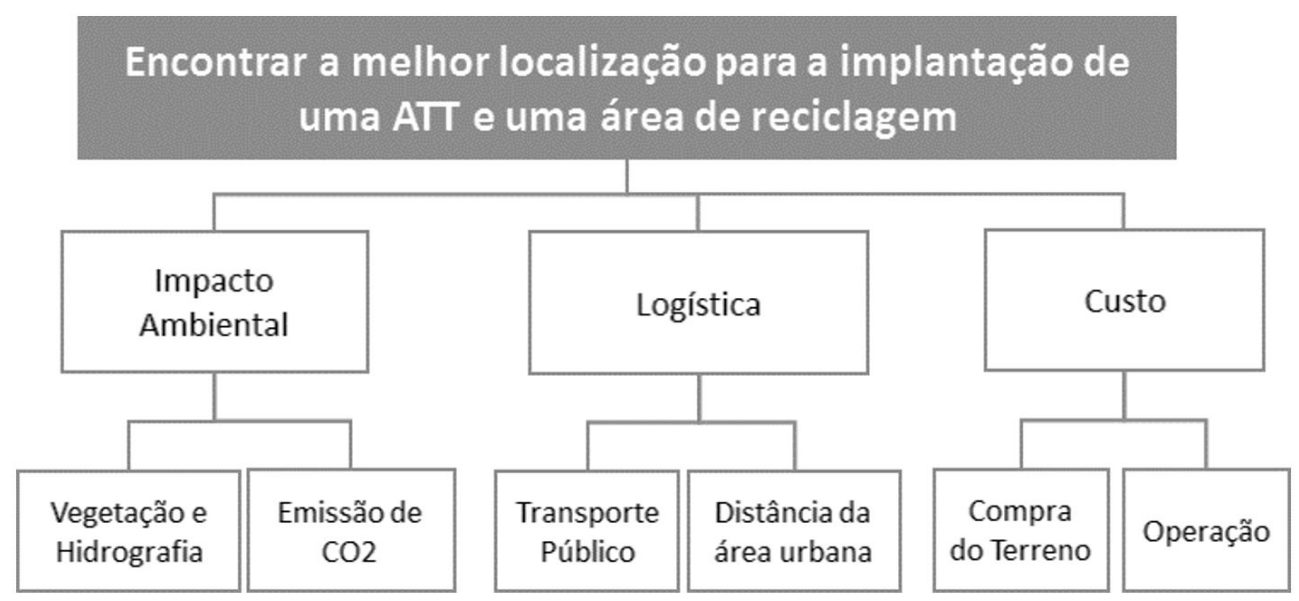

Figura 3. Árvore de valor do modelo. 


\subsection{Passo 3: Identificação das Alternativas}

A ATT e a usina de reciclagem devem atender as grandes construtoras, sendo que estas não possuem um modelo padrão. Existem também obras, como reforma e demolição nos centros das cidades, assim como a expansão e construção de novos empreendimentos nas regiões mais afastadas da cidade. Deste modo, optou-se por definir o local mais apropriado para a implantação da usina, atendendo a fatores gerais, externos à um modelo de empreendimento único. As alternativas foram:

- Proximidade ao centro da cidade mais desenvolvida;

- Afastado do centro da cidade e mais próximo a periferia;

- Próximo a uma via de acesso rápido tanto para o principal centro da cidade como para as outras cidades;

- Localização na interseção dos centros urbanos.

- Para cada tipo de alternativa existem critérios e restrições que ponderam o valor para cada localização. As alternativas foram definidas como sendo a determinada localização em cada bairro da região metropolitana de Belém, Pará.

\subsection{Passo 4: Construção da Função de Valor}

As funções de valor representam a performance das alternativas com relação a um dado critério ou restrição indicado pelo decisor. Foram consideradas escalas de I a 100 onde I representa a pior situação e I00 a melhor dentro de cada critério analisado. As escalas para os atributos estão descritas a seguir.

\subsection{Compra do terreno}

A compra do terreno é um critério (Figura 4) que sintetiza a necessidade de posicionar a planta de tratamento em uma área estratégica visando uma concentração de RCC com custos minimizado. Para tanto, foram consideradas a logística de recebimento dos resíduos, facilidade de instalação de infraestrutura, além da questão social do transporte de colaboradores para a usina e distúrbio para uma vizinhança.

\subsubsection{Transporte público}

Importante considerar a acessibilidade de seus colaboradores, considerando o transporte público para o acesso ao trabalho, conforme critério descrito na Figura 5.

\subsubsection{Distância da área urbana}

Para tentar solucionar o problema dos RCC, a ATT e a usina devem ficar o mais próximo dos grandes centros, nesses locais ocorrem a troca de pavimentos, demolições e a renovação de imóveis. Assim como, as construtoras são grandes geradoras de resíduos e nesses locais ocorrem em grandes quantidades o descarte deste tipo de resíduo. A Figura 6 mostra o critério empregado para este item.

Facilidade de programar logística de captação de RCC, terreno grande com mínimo 100 impacto ambiental, com vias largas e de acesso bem sinalizado, com área interna com possibilidade de expansão. Valor do terreno baixo e poucas áreas vizinhas residenciais.

1 Difícil acesso, estradas estreitas, terreno pequeno e com mananciais e vegetação, valor do terreno alto e maior parte da vizinhança é residencial.

Figura 4. Critério - Compra do Terreno.

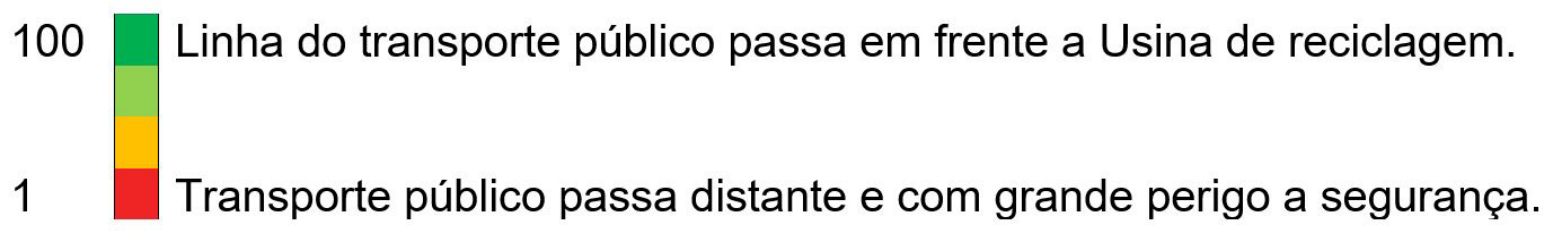

Figura 5. Critério - Transporte Público.

100 Facilidade de obtenção de resíduo, desenvolvimento da produção, reposição de peças e capacitação de colaboradores.

1 Difícil acesso aos resíduos, as peças de reposição e capacitação

Figura 6. Critério - Distância da Área Urbana. 


\subsubsection{Operação}

Custo de operação é um critério (Figura 7) muito significativo no processo de decisão, onde o custo recorrente como a manutenção da usina e dos equipamentos, assim como o custo com insumos e o aluguel varia conforme a localização.

\subsubsection{Emissão de $\mathrm{CO}_{2}$}

A emissão de $\mathrm{CO}_{2}$ está foi analisada de maneira qualitativa onde considera o tempo gasto para a coleta do resíduo, devendo priorizar o tempo ativo no recolhimento de RCCs, sem transporte com carga vazia (Figura 8).

\subsubsection{Vegetação e hidrografia}

A Figura 9 apresenta o critério Vegetação e Hidrografia. Essa variável é substancialmente significativa, pois para a implementação, considerando que as soluções passam por escolhas de tecnologia, é necessário a preparação de editais, a obtenção de licenças, o desenvolvimento de um projeto detalhado, a obtenção ou designação de áreas e acompanhamento de obras. Em todas essas etapas há a fiscalização de órgãos ambientais, Tribunal de Contas, população, etc. A preparação de um bom projeto de tratamento de resíduos sólidos é um investimento cujos resultados não são imediatos, e sempre buscando estar longe de mananciais aquíferos e vegetação densa. Quanto melhor a visão sistêmica, mais transparente frente aos agentes de fiscalização e maior o marketing da unidade de tratamento.

\subsection{Passo 5: Avaliação Parcial das Alternativas}

A construção dos valores para as alternativas foi realizada através de uma estimativa da média dos valores de venda e do tamanho em " $m^{2}$ " dos imóveis em cada bairro, conforme mostrado na Tabela I.

\subsection{Passo 6: Definição dos Pesos dos Atributos}

Para isso, é preciso normalizar os pesos para serem trabalhados no próximo passo, conforme as notas dadas pelo decisor. A Tabela 2 apresenta os pesos de maneira a representar as informações que o decisor achou mais relevante.

Facilidade de programar logística de captação de RCC, terreno com mínimo impacto ambiental e menor valor de aluguel, com vias largas e de acesso bem sinalizado, com área interna com possibilidade de expansão e pouca vizinhança residencial.

Difícil acesso, estradas estreitas, terreno pequeno e com mananciais e vegetação, custo com o aluguel alto e vizinhança residencial.

Figura 7. Critério - Operação. do RCC.

Frota de caminhão sem manutenção, despejo de rejeitos da reciclagem no ambiente, destruição da vegetação e mananciais pela falta de cuidado. Vias estreitas, transito lento, difícil definir uma rota adequada.

Figura 8. Critério - Emissão de $\mathrm{CO}_{2}$. espécies raras.

1 Área próximas de Rios, Vegetação nativa e com espécies raras.

Figura 9. Critério - Vegetação e Hidrografia.

Tabela I. Localização e suas respectivas áreas com valor do aluguel e de compra

\begin{tabular}{|c|c|c|c|}
\hline Localização & Área $\left(\mathrm{m}^{2}\right)$ & Aluguel $\left(\mathrm{R} \$ \times 10^{\wedge} 3\right)$ & Compra $\left(R \$ \times 10^{\wedge} 6\right)$ \\
\hline A - Jurunas & 13200 & 19,8 & 20 \\
\hline B - Umarizal & 10416 & 15,6 & 62 \\
\hline C - Curió-Utinga & 15846 & 23,7 & II \\
\hline D - São Brás & 16000 & 24,0 & 16 \\
\hline E - Souza & 21600 & 32,4 & 62 \\
\hline F - Ananindeua & 10000 & 15,0 & 8 \\
\hline
\end{tabular}


Os atributos foram quantificados segundo critérios do decisor e transformados em uma escala de I a 100.

\subsection{Passo 7: Avaliação Global das Alternativas}

Para a avaliação global das alternativas tem-se $\mathrm{V}(\mathrm{a}) \mathrm{o}$ valor associado à alternativa $a, v_{i}(a)$ a pontuação da alternativa a no i-ésimo critério e $w_{i}$ o peso atribuído a este mostrado na Equação I:

$$
V(a)=\sum_{i=1}^{m} w_{i} v_{i}(a)
$$

Através dos valores atribuídos para cada alternativa (como visto na Tabela 3) entre os pesos gerados e os valores das notas da Tabela 2, foram gerados valores que caracterizam o ranking de cada alternativa (mostrada na Tabela 4).

A localização mais apropriada para a implantação da ATT e Usina de Reciclagem foi a localização D (São Brás) seguida da $F$ (Ananindeua), como mostrado na Tabela 4.

Pela pontuação global, o bairro da São Brás foi a alternativa que melhor atende aos critérios do decisor.

\subsection{Passo 8: Avaliação da Decisão}

Contudo, é preciso analisar se este resultado é de fato válido. Para tanto, construiu-se o gráfico, apresentado na Figura 10, de viabilidade versus custo, os quais são levados como valores o peso da compra do terreno e de operação que são dois ramos da árvore de valor. Para o atributo compra do terreno foi considerado a compra ou o aluguel do terreno

De acordo com o eixo de viabilidade do gráfico da Figura 10 é possível identificar que a alternativa mais viável é a "D” - São Brás. Contudo, esta é uma das opções com maior custo, sendo que a opção "A" - Jurunas apresenta o menor custo estimado.

Outra avaliação realizada foi do critério viabilidade versus transporte público e distância da área urbana como mostrado na Figura II.

Também neste quesito, a opção “D” da Figura I I mostrou ser a mais viável, principalmente, devido as possibilidades de criação de rotas estratégias para buscar o RCC e de transporte dos colaboradores até a unidade.

Tabela 2. Atribuição de valores e o peso para cada atributo

\begin{tabular}{lccccccc}
\hline & $\begin{array}{c}\text { Compra do } \\
\text { Terreno }\end{array}$ & $\begin{array}{c}\text { Vegetação e } \\
\text { Hidrografia }\end{array}$ & Operação & $\begin{array}{c}\text { Distância da } \\
\text { área urbana }\end{array}$ & $\begin{array}{c}\text { Emissão de } \\
\text { CO }_{2}\end{array}$ & $\begin{array}{c}\text { Transporte } \\
\text { Público }\end{array}$ \\
\hline Peso Original & 100 & 82 & 64 & 46 & 28 & 10 & 330 \\
Peso & 30 & 25 & 19 & 14 & 8 & 3 & 100 \\
\hline
\end{tabular}

Tabela 3. Atribuição de valores e o peso para cada atributo

\begin{tabular}{lccccccc}
\hline & Peso & A & B & C & D & E & F \\
\hline Compra do Terreno & 30 & 70 & 40 & 80 & 80 & 50 & 90 \\
Vegetação e Hidrografia & 25 & 50 & 80 & 70 & 90 & 60 & 90 \\
Operação & 19 & 90 & 80 & 90 & 90 & 80 & 90 \\
Distância da área urbana & 14 & 100 & 90 & 80 & 100 & 80 & 70 \\
Emissão de CO & 8 & 50 & 60 & 60 & 80 & 60 & 50 \\
Transporte Público & 3 & 80 & 80 & 60 & 100 & 80 & 100 \\
& & $\mathbf{7 1 7 0}$ & $\mathbf{6 7 5 8}$ & $\mathbf{7 7 1 5}$ & $\mathbf{8 7 8 2}$ & $\mathbf{6 4 2 4}$ & $\mathbf{8 4 1 2}$ \\
\hline
\end{tabular}

Tabela 4. Localização mais apropriada para a implantação da ATT e Usina de Reciclagem

\begin{tabular}{cccccc}
\hline $\mathbf{D}$ & F & C & A & B & E \\
\hline 8782 & 8412 & 7715 & 7170 & 6758 & 6424 \\
\hline
\end{tabular}

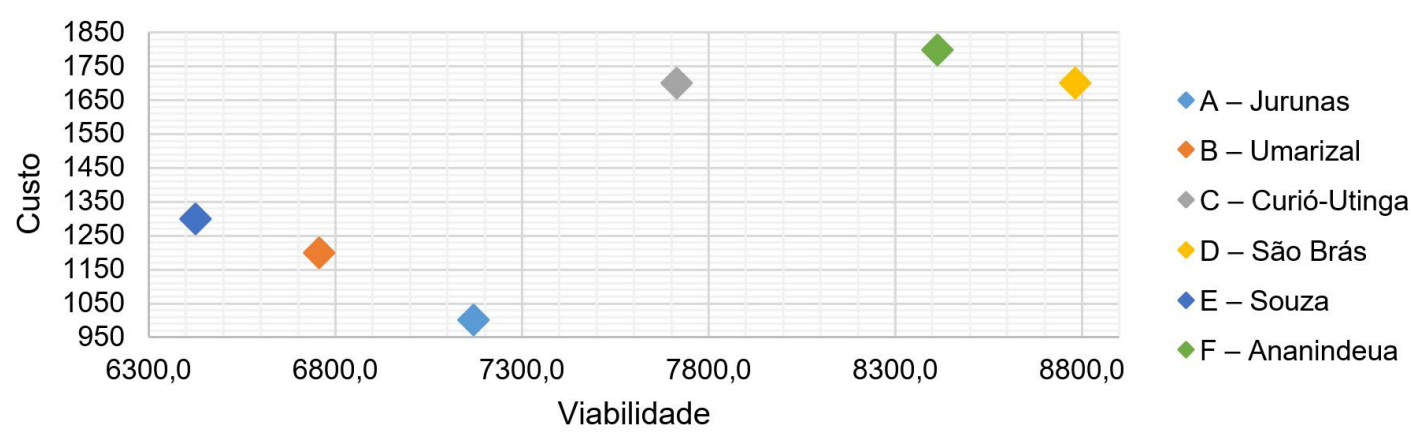

Figura 10. Viabilidade $\times$ custo para as alternativas. 
$\mathrm{Na}$ avaliação viabilidade $\mathrm{x}$ impacto ambiental (Figura I2) a melhor opção foi a alternativa “D”, pois seria um local com a vegetação suprimida e longe de rios, além de possuir a sua localização com boa acessibilidade.

\subsection{Passo 9: Realização da Análise de Sensibilidade}

Devido a diferença entre os pesos para os dados a viabilidade e custo, viabilidade e transporte público e viabilidade e impacto ambientais, realizou-se a análise de sensibilidade para verificar como a pontuação global das alternativas se comporta com a alteração destes valores. Variando os pesos das alternativas para os critérios que representam o custo, obteve-se uma pontuação global como mostrado na Figura 13, percebe-se que através do gráfico, a alternativa $D$ (São Brás) ainda é a mais viável, em seguida, segue a opção $F$ (Ananindeua).

Para a análise de sensibilidade da logística, foi construído o gráfico mostrado na Figura 14. Neste, é possível observar que para os pesos diferentes entre zero e 100, a opção D (São Brás) foi ainda a melhor pontuada.

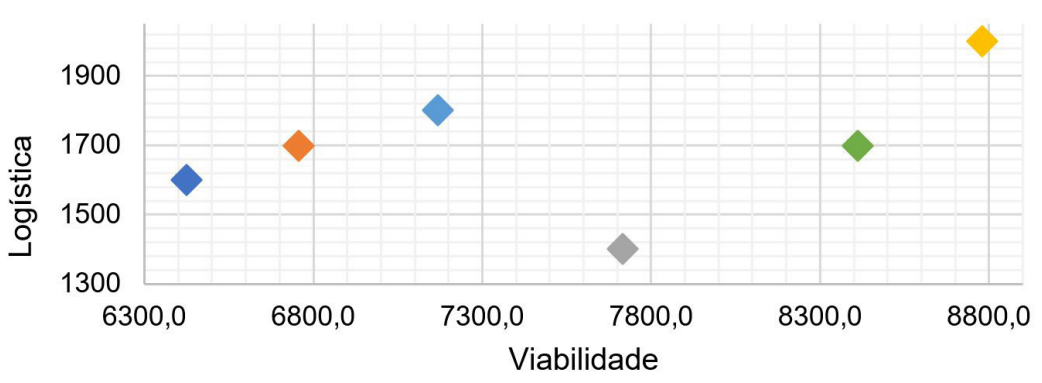

$$
\begin{aligned}
& \text { A - Jurunas } \\
& \text { B - Umarizal } \\
& \text { C - Curió-Utinga } \\
& \text { D - São Brás } \\
& \text { E - Souza } \\
& \text { F - Ananindeua }
\end{aligned}
$$

Figura I I. Viabilidade $\times$ Logística para as alternativas.

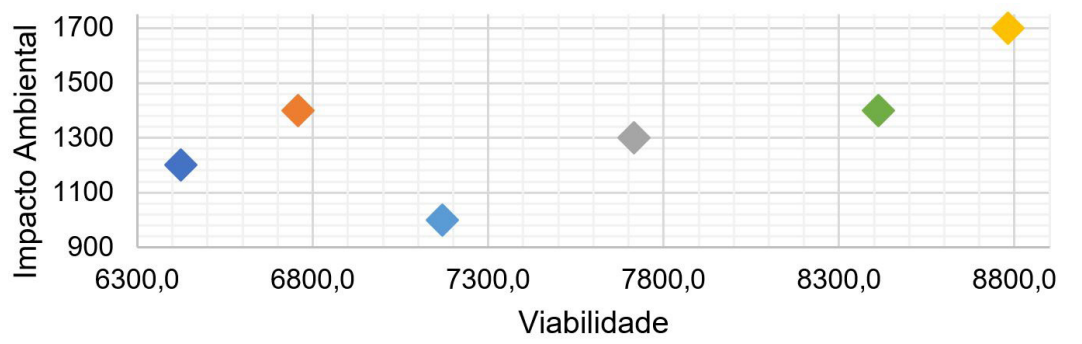

$$
\begin{aligned}
& \text { A - Jurunas } \\
& \text { B - Umarizal } \\
& \text { C - Curió-Utinga } \\
& \text { D - São Brás } \\
& \text { E - Souza } \\
& \text { F - Ananindeua }
\end{aligned}
$$

Figura I2. Viabilidade $\times$ Impacto Ambiental para as alternativas.

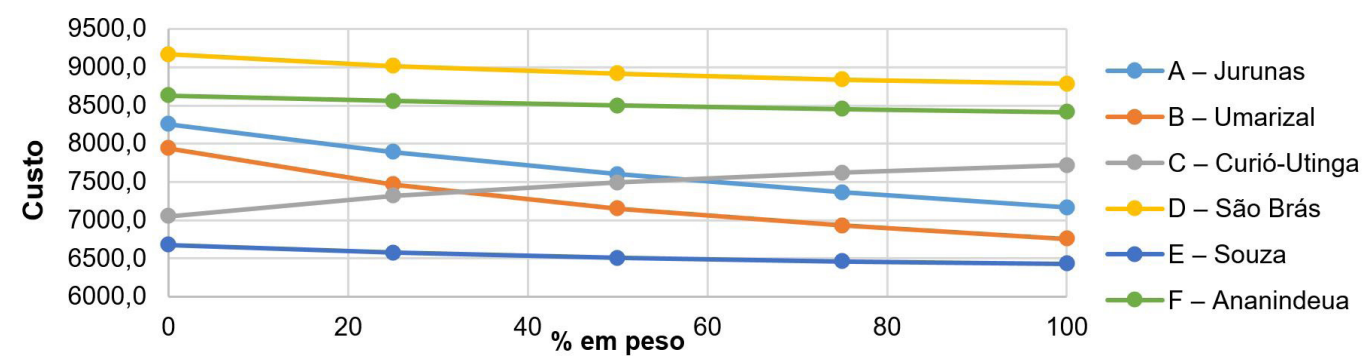

Figura 13. Análise de sensibilidade para o custo dentre as alternativas.

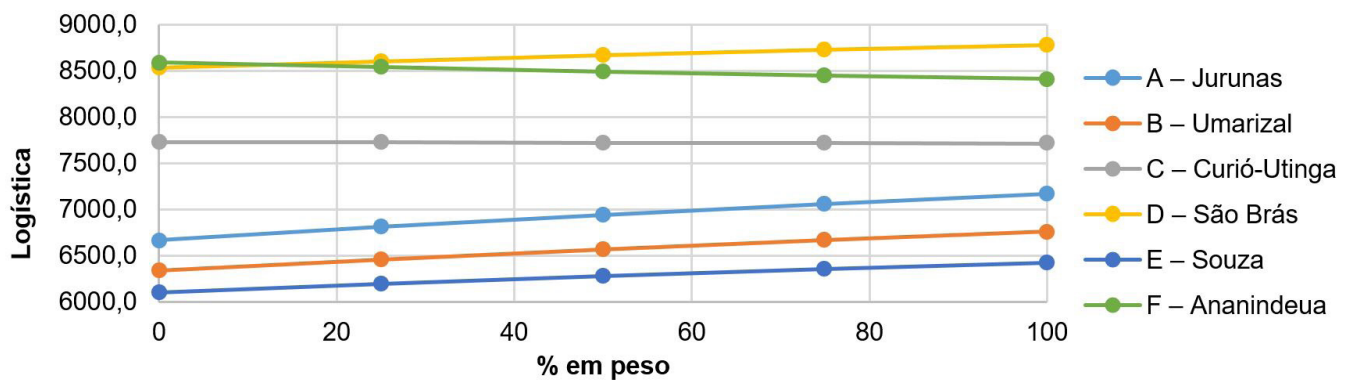

Figura 14. Análise de sensibilidade para a logística dentre as alternativas. 


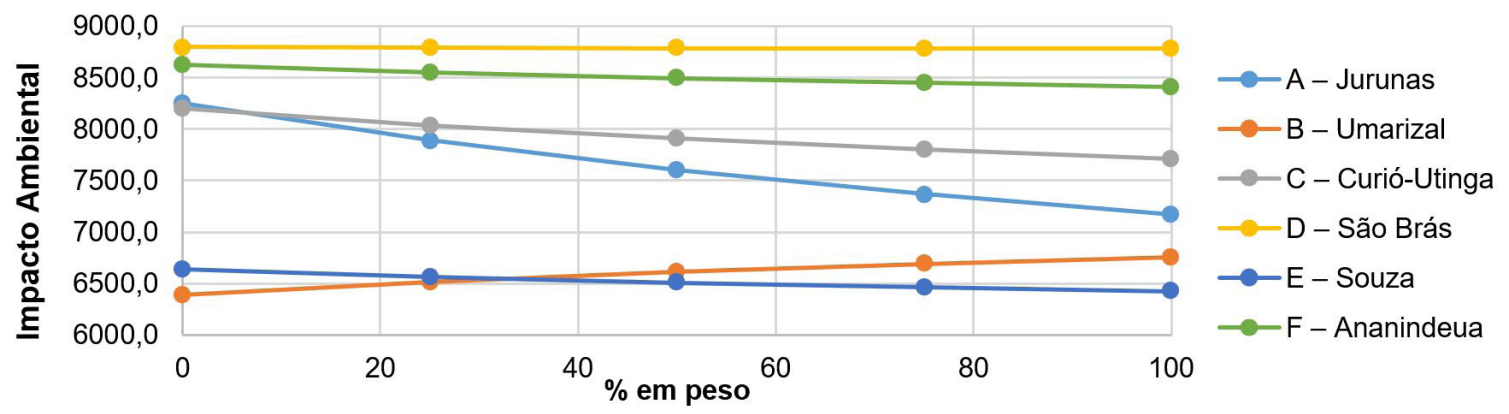

Figura I5. Análise de sensibilidade para o impacto ambiental dentre as alternativas.

Para o critério impacto ambiental foi realizado a análise de sensibilidade (Figura I5), no qual a alternativa D (São Brás) é a melhor pontuada, mesmo com o peso igual a zero, em seguida a alternativa $F$ (Ananindeua).

\section{CONCLUSÃO}

A alternativa selecionada foi o Bairro de São Brás. O que diferenciou das outras alternativas, é que ganha nos critérios de maior peso, o terreno que será ocupado atingirá o mínimo de área com vegetação e manancial, além de possibilitar a chegada dos colaborados através do transporte público e facilitar a logística dos resíduos. Uma análise mais detalhada pode também ser considerada para futuros estudos, considerando separadamente cenários que abordam a responsabilidade sobre a coleta do RCC pelo construtor, a responsabilidade sobre a coleta de RCC transferida do construtor para a usina, um possível convênio entre o estado/municípios/construtoras, bem como uma validação das rotas dos veículos perante o gasto quantitativo de emissões de $\mathrm{CO}_{2}$.

\section{REFERÊNCIAS}

I Alves JO, Junca E, Espinosa DCR, Tenório JAS. Resíduo do corte de granito: inovação tecnológica para a destinação final. Tecnologica em Metalurgia, Materiais e Mineração. 2015; I2(2): I23-I 28.

2 Shinomiya LD, Gomes JO, Alves JO. Planejamento de cenários para uso de resíduos industriais: aplicação para lama vermelha. Revista Gestão em Engenharia. 2015;2:43-66.

3 Brasil. Ministério do Meio Ambiente - MMA. Lei $n^{\circ}$ 12.305, de 2 de agosto de 20 I0. Política Nacional de Resíduos Sólidos. Diário Oficial da República Federativa do Brasil. 2010 Ago. 3.

4 Manzini E, Vezzoli C. O desenvolvimento de produtos sustentáveis. Vol. I. São Paulo: EDUSP; 2008.

5 Associação Brasileira de Normas Técnicas. ABNT NBR n 15. I | 2c: resíduos da construção civil e resíduos volumosos: áreas de transbordo e triagem: diretrizes para projeto, implantação e operação. Rio de Janeiro: ABNT; 2004.

6 Associação Brasileira de Normas Técnicas. ABNT NBR n 15. I 4a: Resíduos sólidos da construção civil: diretrizes para projeto, implantação e operação. Rio de Janeiro: ABNT; 2004.

7 Zhuo C, Alves JO, Tenório JAS, Levendis YA. Synthesis of carbon nanomaterials through up-cycling agricultural and municipal solid wastes. Industrial \& Engineering Chemistry Research. 20I2;5 I(7):2922-2930. http://dx.doi. org/I0.102I/ie2027IIh.

8 Franco LA, Montibeller G. Facilitated modelling in operational research. European Journal of Operational Research. 20I0;205(3):489-500.

9 Lachtermacher G. Pesquisa operacional na tomada de decisões: para cursos de administração, economia e ciências contábeis. 2. ed. Rio de Janeiro: Campus; 2004.

Recebido em: 13 Set. 2018

Aceito em: 15 Fev. 2019 